\title{
INTERIOR POINTS OF THE COMPLETELY POSITIVE CONE*
}

\author{
MIRJAM DÜR ${ }^{\dagger}$ AND GEORG STILL ${ }^{\ddagger}$
}

\begin{abstract}
A matrix $A$ is called completely positive if it can be decomposed as $A=B B^{T}$ with an entrywise nonnegative matrix $B$. The set of all such matrices is a convex cone which plays a role in certain optimization problems. A characterization of the interior of this cone is provided.
\end{abstract}

Key words. Completely positive matrices, Copositive matrices, Cones of matrices.

AMS subject classifications. 15A23, 15A48.

1. Introduction. A symmetric matrix $A$ is called completely positive if it allows a factorization $A=B B^{T}$ with an entrywise nonnegative matrix $B$. This class of matrices has received quite an amount of interest in the linear algebra literature during the last decades. An excellent survey of this literature is the book [2]. However, completely positive matrices have recently also attracted some interest in the mathematical programming community.

Since the 1980s, so called semidefinite relaxations have been proposed as a strong method to obtain good bounds for many combinatorial optimization problems. A semidefinite program is an optimization problem where a linear function of a matrix variable is to be minimized subject to linear constraints and an additional semidefiniteness constraint, i.e., one wants to optimize over the cone $\mathcal{P}$ of positive semidefinite matrices. Efficient algorithms called interior point methods have been developed for this type of problem. For an introduction to semidefinite programming, see [7].

Starting with [3], it has then been observed that some combinatorial problems like the maximum clique problem can equivalently be reformulated as optimization problems over the cone $\mathcal{C P}$ of completely positive matrices. Burer [4] showed the very general result that every quadratic problem with linear and binary constraints can be rewritten as such a problem. More precisely, he showed that a quadratic binary

${ }^{*}$ Received by the editors October 06, 2007. Accepted for publication January 20, 2008. Handling Editor: Raphael Loewy.

†Technische Universität Darmstadt, Department of Mathematics, Schloßgartenstr. 7, 64289 Darmstadt, Germany (duer@mathematik.tu-darmstadt.de).

${ }^{\ddagger}$ University of Twente, Department of Applied Mathematics, P.O.Box 217, 7500 AE Enschede, The Netherlands (g.still@math.utwente.nl). 
problem of the form

$$
\begin{array}{cl}
\min & x^{T} Q x+2 c^{T} x \\
\text { s.t. } & a_{i}^{T} x=b_{i} \quad(i=1, \ldots, m) \\
& x \geq 0 \\
& x_{j} \in\{0,1\} \quad(j \in B)
\end{array}
$$

(with $Q$ not necessarily positive semidefinite) can equivalently be written as the following linear problem over the cone of completely positive matrices:

$$
\begin{array}{cl}
\min & \langle Q, X\rangle+2 c^{T} x \\
\mathrm{s.t.} & a_{i}^{T} x=b_{i} \quad(i=1, \ldots, m) \\
& \left\langle a_{i} a_{i}^{T}, X\right\rangle=b_{i}^{2} \quad(i=1, \ldots, m) \\
& x_{j}=X_{j j} \quad(j \in B) \\
& \left(\begin{array}{ll}
1 & x \\
x & X
\end{array}\right) \in \mathcal{C P} .
\end{array}
$$

This is a remarkable result, since it transforms a nonconvex quadratic integer problem equivalently into a linear problem over a convex cone, i.e., a convex optimization problem which has no nonglobal local optima. The difficulty, of course, is now in the cone constraint, whence it is essential to get a better understanding of the cone.

The dual problem of a completely positive program is an optimization problem over the cone of copositive matrices. Obviously, both problem classes are NP-hard since they are equivalent to integer programming.

Interior point algorithms have proved to be very efficient for semidefinite problems. Because of the nonpolynomial complexity of completely positive programs (in contrast to polynomial complexity of interior point methods for semidefinite programs) it will not be possible to extend these methods directly to the completely positive cone. But one might still try to design algorithms which use interior points for a completely positive program. However, nothing seems to be known about the structure of the interior of $\mathcal{C P}$. This is what we investigate in this note.

We use the following notation:

$\mathcal{S}=\left\{A \in \mathbb{R}^{n \times n}: A=A^{T}\right\}$, the cone of symmetric matrices,

$\mathcal{N}=\{A \in \mathcal{S}: A \geq 0\}$, the cone of (entrywise) nonnegative matrices,

$\mathcal{P}=\{A \in \mathcal{S}: A \succeq 0\}$, the cone of positive semidefinite matrices,

$\mathcal{C P}=\left\{B=\sum_{i=1}^{m} a_{i} a_{i}^{T}: a_{i} \geq 0\right\}$, the cone of completely positive matrices,

$\mathcal{C O P}=\left\{A \in \mathcal{S}: x^{T} A x \geq 0 \forall x \geq 0\right\}$, the cone of copositive matrices. 
An equivalent definition is $\mathcal{C P}=\left\{B=A A^{T}: A \in \mathbb{R}^{n \times m}, A \geq 0\right\}$. Clearly, the factorization of a completely positive matrix is not unique.

Obviously, we have the following relations:

$$
\mathcal{C P} \subseteq \mathcal{P} \cap \mathcal{N} \text { and } \mathcal{C O P} \supseteq \mathcal{P}+\mathcal{N}
$$

Interestingly, for $n \times n$-matrices of order $n \leq 4$, we have equality in the above relations, whereas for $n \geq 5$, both inclusions are strict, cf. [6].

The inner product in $\mathcal{S}$ is defined as $\langle A, B\rangle:=\operatorname{trace}(A B)$. For a given cone $\mathcal{K} \subseteq \mathcal{S}$, the dual cone $\mathcal{K}^{*}$ is defined as

$$
\mathcal{K}^{*}:=\{A \in \mathcal{S}:\langle A, B\rangle \geq 0 \text { for all } B \in \mathcal{K}\} .
$$

It can be shown that $\mathcal{K}=\left(\mathcal{K}^{*}\right)^{*}$ if and only if $\mathcal{K}$ is a closed convex cone (see for example [2, Theorem 1.36]). All matrix cones defined above are closed convex cones. We have

$$
\mathcal{S}^{*}=\{0\}, \quad \mathcal{N}^{*}=\mathcal{N}, \quad \mathcal{P}^{*}=\mathcal{P}, \quad \mathcal{C} \mathcal{P}^{*}=\mathcal{C O P}, \quad \mathcal{C O} \mathcal{P}^{*}=\mathcal{C P} .
$$

For a proof of the last two relations see for instance [2, Theorem 2.3].

It is easy to see that $\operatorname{int}(\mathcal{N})=\{A \in \mathcal{S}: A>0\}$ and $\operatorname{int}(\mathcal{P})=\{A \in \mathcal{S}: A \succ 0\}=$ $\left\{A=B B^{T}: B \in \mathbb{R}^{n \times n}\right.$ nonsingular $\}$. For the dual of a closed convex cone $\mathcal{K}$, it has been shown in $[1$, Chapter 1 , Section 2] that

$$
\operatorname{int}\left(\mathcal{K}^{*}\right)=\{A \in \mathcal{S}:\langle A, B\rangle>0 \text { for all } B \in \mathcal{K} \backslash\{0\}\} .
$$

From this relation, it is not difficult to derive a characterization of the interior of $\mathcal{C O P}$ : we get

$$
\operatorname{int}(\mathcal{C O P})=\left\{A \in \mathcal{S}: x^{T} A x>0 \text { for all } x \geq 0, x \neq 0\right\},
$$

which says that the interior of $\mathcal{C O P}$ consists of the so called strictly copositive matrices.

This is a well known result, but as far as we are aware, no analogous result is known for the cone $\mathcal{C P}$. The next section provides a characterization of its interior.

2. Characterization of the Interior of $\mathcal{C P}$. It follows from the definition of $\mathcal{C P}$ that the inclusion

$$
\mathcal{C P} \subseteq \mathcal{P} \cap \mathcal{N}
$$

always holds. From this, we directly conclude

$$
\operatorname{int}(\mathcal{C P}) \subseteq \operatorname{int}(\mathcal{P}) \cap \operatorname{int}(\mathcal{N})=\{A \in \mathcal{S}: A \succ 0, A>0\}
$$


which immediately implies the following:

Lemma 2.1. If $A \in \operatorname{int}(\mathcal{C P})$, then $A>0$ and $\operatorname{rank} A=n$.

Moreover, since for $n \leq 4$ equality holds in (2.1), we must have

$$
\operatorname{int}(\mathcal{C P})=\{A \in \mathcal{S}: A \succ 0, A>0\} \quad \text { for } n \leq 4 .
$$

On the other hand, the fact that for $n \geq 5$ the inclusion (2.1) is strict implies that also the inclusion (2.2) is strict for $n \geq 5$. Indeed, if we choose matrices $A \in(\mathcal{P} \cap \mathcal{N}) \backslash \mathcal{C P}$ and $B \in \operatorname{int}(\mathcal{P} \cap \mathcal{N})$, then by convexity,

$$
X_{\lambda}:=A+\lambda(B-A) \in \operatorname{int}(\mathcal{P} \cap \mathcal{N}) \text { for all } 0<\lambda \leq 1
$$

However, since $\mathcal{C P}$ is closed, the relation $A \notin \mathcal{C P}$ implies $X_{\lambda} \notin \mathcal{C P}$ for $\lambda>0$ small enough, so that $X_{\lambda} \in \operatorname{int}(\mathcal{P} \cap \mathcal{N}) \backslash \mathcal{C P}$. We also provide a concrete counterexample.

Example 2.2. Take

$$
A=\left(\begin{array}{llllllllll}
1 & 0 & 0 & 0 & 1 & 1 & 0 & 0 & 1 & 2 \\
1 & 1 & 0 & 0 & 0 & 2 & 1 & 0 & 0 & 1 \\
0 & 1 & 1 & 0 & 0 & 1 & 2 & 1 & 0 & 0 \\
0 & 0 & 1 & 1 & 0 & 0 & 1 & 2 & 1 & 0 \\
0 & 0 & 0 & 1 & 1 & 0 & 0 & 1 & 2 & 1
\end{array}\right) \quad \Rightarrow \quad A A^{T}=\left(\begin{array}{lllll}
8 & 5 & 1 & 1 & 5 \\
5 & 8 & 5 & 1 & 1 \\
1 & 5 & 8 & 5 & 1 \\
1 & 1 & 5 & 8 & 5 \\
5 & 1 & 1 & 5 & 8
\end{array}\right) .
$$

Clearly, $A A^{T}>0$ and $\operatorname{rank} A A^{T}=5$, so $A A^{T} \in \operatorname{int}(\mathcal{P} \cap \mathcal{N})$. Nevertheless, there exists a copositive matrix $H$ such that $\left\langle A A^{T}, H\right\rangle=0$, which, by (1.1), proves that $A A^{T} \notin \operatorname{int}(\mathcal{C P})$. This matrix is the Horn matrix

$$
H=\left(\begin{array}{rrrrr}
1 & -1 & 1 & 1 & -1 \\
-1 & 1 & -1 & 1 & 1 \\
1 & -1 & 1 & -1 & 1 \\
1 & 1 & -1 & 1 & -1 \\
-1 & 1 & 1 & -1 & 1
\end{array}\right)
$$

which was introduced by Horn to illustrate that there exist copositive matrices which are not decomposable as the sum of a positive semidefinite and a nonnegative matrix, cf. [5]. To see that $H$ is copositive, write

$$
\begin{aligned}
x^{T} H x & =\left(x_{1}-x_{2}+x_{3}+x_{4}-x_{5}\right)^{2}+4 x_{2} x_{4}+4 x_{3}\left(x_{5}-x_{4}\right) \\
& =\left(x_{1}-x_{2}+x_{3}-x_{4}+x_{5}\right)^{2}+4 x_{2} x_{5}+4 x_{1}\left(x_{4}-x_{5}\right) .
\end{aligned}
$$

The first expression shows that $x^{T} H x \geq 0$ for nonnegative $x$ with $x_{5} \geq x_{4}$, whereas the second expression shows $x^{T} H x \geq 0$ for nonnegative $x$ with $x_{5}<x_{4}$. 
In view of the preceding discussions, to find a characterization of $\operatorname{int}(\mathcal{C P})$ for arbitrary matrix dimensions we have to look for subsets of the cone

$$
\left\{A A^{T}: A A^{T}>0, A \geq 0, \operatorname{rank} A A^{T}=n\right\} .
$$

The next theorem gives such a characterization. We use the notation $\left[A_{1} \mid A_{2}\right]$ to describe the matrix whose columns are the columns of $A_{1}$ augmented with the columns of $A_{2}$.

TheOREM 2.3. We have:

$$
\operatorname{int}(\mathcal{C P})=\left\{A A^{T}: A=\left[A_{1} \mid A_{2}\right] \text { with } A_{1}>0 \text { nonsingular, } A_{2} \geq 0\right\}
$$

Proof. Denote $\mathcal{M}:=\left\{A A^{T}: A=\left[A_{1} \mid A_{2}\right]\right.$ with $A_{1}>0$ nonsingular, $\left.A_{2} \geq 0\right\}$ for abbreviation.

$[\mathcal{M} \subseteq \operatorname{int}(\mathcal{C P})]:$ Let $B \in \mathcal{M}$. We show that $B_{1}=A_{1} A_{1}^{T} \in \operatorname{int}(\mathcal{C P})$. Then, since $\mathcal{C P}$ is a cone, also $B=A_{1} A_{1}^{T}+A_{2} A_{2}^{T} \in \operatorname{int}(\mathcal{C P})$.

So we only have to show that the statement holds for $B=A A^{T}$ with nonsingular $0<A \in \mathbb{R}^{n \times n}$. To do so, we choose $S \in \mathcal{S}$ arbitrarily and prove that for any $\varepsilon$ small enough there exists $C \in \mathbb{R}^{n \times n}, C \geq 0$, such that

$$
A A^{T}+\varepsilon S=C C^{T} .
$$

The relation (2.3) is equivalent to

$$
I+\varepsilon A^{-1} S A^{-T}=\left(A^{-1} C\right)\left(A^{-1} C\right)^{T} .
$$

We put $M:=A^{-1} S A^{-T}$ and note that for small $\varepsilon$ the matrix $I+\varepsilon M$ is positive definite. It is well-known that by a (symmetric) Gauss elimination algorithm (e.g. the Cholesky decomposition) any positive definite matrix $E$ can be decomposed as

$$
E=Q Q^{T} \text { with nonsingular } Q .
$$

This transformation $Q=Q(E)$ depends continuously on $E$. Therefore, since $I$ has the obvious decomposition $I=Q Q^{T}$ with $Q(I)=I$, also $I+\varepsilon M$ has a decomposition

$$
I+\varepsilon M=Q Q^{T}, \quad Q=Q(I+\varepsilon M)=I+V(\varepsilon),
$$

with some matrix $V(\varepsilon)$ which tends to the zero matrix as $\varepsilon \rightarrow 0$. Comparing with (2.4), we can put $A^{-1} C=Q$ and we finally obtain a representation (2.3) with

$$
C=A Q=A(I+V(\varepsilon))=A+A V(\varepsilon)>0
$$


for all small $\varepsilon$, which proves $\mathcal{M} \subseteq \operatorname{int}(\mathcal{C P})$.

$[\operatorname{int}(\mathcal{C P}) \subseteq \mathcal{M}]:$ Let $B$ be an arbitrary matrix in $\operatorname{int}(\mathcal{C P})$, and choose some matrix

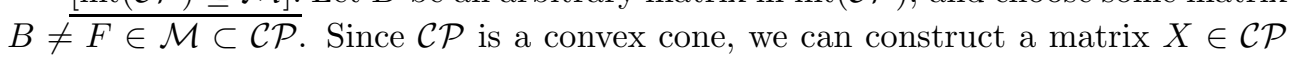
such that $B$ is a strict convex combination of $F$ and $X$. Indeed, since $B \in \operatorname{int}(\mathcal{C P})$, there exists $\alpha>1$ such that $X:=F+\alpha(B-F) \in \mathcal{C P}$. The last equation is equivalent to

$$
B=\left(1-\frac{1}{\alpha}\right) F+\frac{1}{\alpha} X=\tilde{F}+\tilde{X}
$$

Clearly, $\tilde{F}=\tilde{A} \tilde{A}^{T}$ with $\tilde{A}=\left[\tilde{A}_{1} \mid \tilde{A}_{2}\right]$ with $\tilde{A}_{1}>0$ nonsingular, $\tilde{A}_{2} \geq 0$, and $\tilde{X}=Y Y^{T}$ for some $Y \geq 0$ since $\tilde{X} \in \mathcal{C P}$. Consequently,

$$
B=\left[\tilde{A}_{1}\left|\tilde{A}_{2}\right| Y\right]\left[\tilde{A}_{1}\left|\tilde{A}_{2}\right| Y\right]^{T}
$$

is a factorization of $B$ with the required properties, whence $B \in \mathcal{M}$.

Acknowledgment. We wish to thank the referee for careful reading and helpful comments.

\section{REFERENCES}

[1] A. Berman. Cones, Matrices and Mathematical Programming. Lecture Notes in Economics and Mathematical Systems 79, Springer Verlag 1973.

[2] A. Berman and N. Shaked-Monderer. Completely Positive Matrices. World Scientific, 2003.

[3] I.M. Bomze, M. Dür, E. de Klerk, C. Roos, A.J. Quist, and T. Terlaky. On Copositive Programming and Standard Quadratic Optimization Problems, Journal of Global Optimization, 18:301-320, 2000.

[4] S. Burer. On the Copositive Representation of Binary and Continuous Nonconvex Quadratic Programs, Preprint, University of Iowa, 2006, available online at http://www.optimization-online.org/DB_HTML/2006/10/1501.html

[5] M. Hall Jr. and M. Newman. Copositive and Completely Positive Quadratic Forms, Proceedings of the Cambridge Philosophical Society, 59:329-33, 1963.

[6] J.E. Maxfield and H. Minc. On the Matrix Equation $X^{\prime} X=A$, Proceedings of the Edinburgh Mathematical Society, 13:125-129, 1962/1963.

[7] H. Wolkowicz (Ed.). Handbook of Semidefinite Programming: Theory, Algorithms, and Applications. Kluwer Academic Publishers, 2000. 